\title{
ON THE CURVATURES OF (k+1)-DIMENSIONAL SEMI-RULED SURFACES IN $E_{v}^{n+1}$
}

\author{
Cumali Ekici \& Ali Görgülü
}

Osmangazi University, Dept. of Mathematics, 26480 Eskişehir-Turkey,

Abstract - In this paper, first we will define the generalized $(k+1)$-dimensional semiruled surface $M^{*}$, such that the generator space of $M^{*}$ is the semi-subspace of $E_{v}^{n+1}$ where $E_{v}^{n+1}$ is the semi-Euclidean space. Then, we will compute the mean curvature, Riemann curvature, Ricci curvature and scalar curvature of $M^{*}$.

\section{INTRODUCTION}

In this section, we will give some preliminaries. We assume that all manifolds, maps, vector fields, etc. ... are differentiable of class $C^{\infty}$.

Let $M$ be a semi-Euclidean submonifold of $E_{v}^{n+1}, \bar{D}$ be a Levi-Civita connection of $E_{y}^{n+1}$ and $D$ be a Levi-Civita connection of $M$. If $X, Y \in \chi(M)$ and $\Pi$ is the second fundamental form of $M$, then we have the Gauss equation

$$
\bar{D}_{X} Y=D_{X} Y+\Pi(X, Y)
$$

[4].

Let $\xi$ be a unit normal vector of $M$. Then, the Weingarten equation is

$$
\bar{D}_{X} \xi=-A_{\xi}(X)+D_{X}^{\perp} \xi
$$

where $A_{\xi}$ determines at each point a self-adjoint linear map on $T_{M}(p)$ and $D^{\perp}$ is a metric connection [3]. We note that, in this paper, $A_{\xi}$ will be used for the linear map and the corresponding matrix of the linear map. 
From equation (1) and (2), it follows

$$
\langle\Pi(X, Y), \xi\rangle=\left\langle A_{\xi}(X), Y\right\rangle
$$

and

$$
\Pi(X, Y)=\sum_{j=1}^{n-m}\left\langle A_{\xi_{j}}(X), Y\right\rangle \xi_{j}
$$

[4].

Let $M$ be an $m$-dimensional semi-Riemannian submanifold in $E_{v}^{n+1}$ and $A_{\xi}$ be a linear map. If $\xi \in \chi^{\perp}(M)$ is a normal unit vector at the point $p \in M$, then

$$
G(p, \xi)=\operatorname{det} A_{\xi}
$$

is called the Lipschitz-Killing vector of $M$ at $p$ in the direction $\xi$ [3].

If $\xi_{1}, \xi_{2}, \ldots, \xi_{n-m}$ constitute an orthonormal basis field of the normal bundle $\chi^{\perp}(M)$, then, the mean curvature $H$ is defined by

$$
H=\sum_{j=1}^{n-m} \frac{\dot{I} z A_{\xi_{j}}}{b o y M} \xi_{j}
$$

[1].

For every $X_{i} \in \chi(M), \quad i=1,2,3,4$ the 4 th order covariant tensor field defined by $R$ as

$$
R\left(X_{1}, X_{2}, X_{3}, X_{4}\right)=\left\langle X_{1}, R\left(X_{3}, X_{4}\right) X_{2}\right\rangle
$$

is called the Riemann curvature tensor field and its value at a point $p \in M$, is called Riemann curvature of $M$ at $p$, where $M$ is an $m$-dimensional semi-Riemannian submanifold in $E_{v}^{n+1}$ [1].

The Riemann curvature is denoted by

$$
K(X, Y)|p=\langle X, R(X, Y) Y\rangle| p
$$

[1]. If $\Pi$ is the second fundamental form, then we have

$$
\langle X, R(X, Y) Y\rangle=\langle\Pi(X, Y), \Pi(Y, Y)\rangle-\langle\Pi(X, Y), \Pi(X, Y)\rangle
$$


Let $M$ be an $m$-dimensional semi-Riemann manifold. A 2-dimensional subspace of $M$ the tangent plane $T_{M}(p)$ of $M$ at $\mathrm{p}$ is called tha tangent plane of $M$ at $\mathrm{p}$ and is denoted by $\mathfrak{I}$. For all $X_{p}, Y_{p} \in \mathfrak{I}$, the real valued function $K$ defined by

$$
K\left(X_{p}, Y_{p}\right)=\frac{\left\langle R\left(X_{p}, Y_{p}\right) Y_{p}, X_{p}\right\rangle}{\left\langle X_{p}, X_{p}\right\rangle\left\langle Y_{p}, Y_{p}\right\rangle-\left\langle X_{p}, Y_{p}\right\rangle^{2}}
$$

is called the sectional curvature function of $M$ at the point $p . K\left(X_{p}, Y_{p}\right)$ is called the sectional curvature of $M$ at $p$ [4].

Let $M$ be an $m$-dimensional semi-Riemann manifold and $R$ be the Riemann curvature tensor. The tensor field $S$ defined in the form

$$
S(X, Y)=\sum_{i=1}^{m} \varepsilon_{i}\left\langle R\left(e_{i}, X\right) Y, e_{i}\right\rangle
$$

is called the Ricci curvature tensor field, where $\left\{e_{1}, e_{2}, \ldots, e_{m}\right\}$ is a system of orthonormal basis of $T_{M}(p)$ and the value of $S(X, Y)$ at $p \in M$ is called the Ricci curvature [4]. Here

$$
\varepsilon_{i}=\left\langle e_{i}, e_{i}\right\rangle, \quad \varepsilon_{i}= \begin{cases}-1 & , \text { if } e_{i} \text { time-like } \\ +1 & , \text { if } e_{i} \text { space-like }\end{cases}
$$

Let $M$ be an $m$-dimensional semi-Riemann manifold and $\left\{e_{1}, e_{2}, . ., e_{m}\right\}$ is an orthonormal basis for $T_{M}(p)$ for $p \in M$. The real number $r_{s k}$ defined in the form

$$
r_{s k}=\sum_{i=1}^{m} S\left(e_{i}, e_{i}\right)
$$

or

$$
r_{s k}=\sum_{i \neq j} K\left(e_{i}, e_{j}\right)=2 \sum_{i<j}^{m} K\left(e_{i}, e_{j}\right)
$$

is called the scaler curvature of $M$ where $S$ is the Ricci curvature tensor field of $M$ [4].

\section{CURVATURES OF SEMI-RULED SURFACES IN $E_{v}^{n+1}$}

In this section, we will calculate the Lipschitz-Killing curvature, section curvature, Ricci curvature, mean curvature and the scalar normal curvature of a semi-ruled surface $M^{*}$ in the semi-Euclidean space $E_{v}^{n+1}$.

Let $\alpha$ be the smooth curve 


$$
\begin{aligned}
\alpha: I & \rightarrow E_{v}^{n+1} \\
t & \rightarrow \alpha(t)=\left(\alpha_{1}(\mathrm{t}), . ., \alpha_{n+1}(t)\right)
\end{aligned}
$$

in the $(n+1)$-dimensional semi-Euclidean space $E_{\mathrm{v}}^{n+1}$ where $\{0\} \subset I \subset \mathfrak{R}$. Let $\left\{e_{1}(\mathrm{t}), e_{2}(\mathrm{t}), \ldots, e_{k}(t)\right\}$ be an orthonormal vector system defined at each $\alpha(t)$ curve $\alpha$. This system spans a subspace of the tangent space $T_{E_{v}^{n+1}}(\alpha(t))$ at $\alpha(t) \in E_{v}^{n+1}$. If this space is shown by $E_{k, \mu}(t)$, then, it is a $k$-dimensional subspace of the form

$$
E_{k, \mu}(t)=S p\left\{e_{1}(\mathrm{t}), e_{2}(\mathrm{t}), . ., e_{k}(t)\right\} \subset E_{v}^{n+1}, 0 \leq \mu \leq v
$$

$E_{k, \mu}(t), \mu \geq 1$, will be called a semi-subspace and satisfied

$$
\left\langle e_{i}(\mathrm{t}), e_{j}(t)\right\rangle=\varepsilon_{i} \delta_{i j}, \quad \varepsilon_{i}= \begin{cases}+1 & , 1 \leq i \leq k-\mu \\ -1 & , k-\mu+1 \leq i \leq k .\end{cases}
$$

For $\mu \geq 1$, there are $\mu$ time-like vectors in the semi-subspace $E_{k, \mu}(t)$. Since there is no time-like vector field on $E_{k, 0}(t)$ for $\mu=0$, then, $E_{k, 0}(t)=E_{k}(t)$ and it is a Euclidean subspace. If $\mu=1$, then, there is one time-like vector, so $E_{k, 1}(t)$ is a time-like subspace.

Throughout this paper, we assume that $E_{k, \mu}(t), \quad \mu \geq 1$, is a semi-subspace.

Definition 1 While the semi subspace $E_{k, \mu}(t)$ moves along a curve $\alpha$ in $E_{v}^{n+1}$, it forms a $(k+1)$-dimensional surface. This surface is called the $(k+1)$-dimensional generalized semi-ruled surface in the $(n+1)$ - dimensional semi-Euclidean space $E_{v}^{n+1}$ and is denoted by $M^{*}$.

Definition $2 E_{k, \mu}(t)$ is called the genarating space at $\alpha(t)$ of the semi-ruled surface $M^{*}$ and the curve $\alpha$ is called the base curve of $M^{*}$.

Let $M^{*}$ be a $(k+1)$-dimensional generalized semi-ruled surface in $E_{\mathrm{v}}^{n+1}$ $E_{k, \mu}(t)=S_{p}\left\{e_{1}(t), \ldots, e_{k}(t)\right\} \subset E_{v}^{n+1}, 0 \leq \mu \leq v$, be the generating space and $\alpha$ be the base curve parametrized by the arc-length. Then, $M^{*}$ can be expressed by the parametric equation 


$$
\varphi\left(t, u_{1}, u_{2}, \ldots, u_{k}\right)=\alpha(t)+\sum_{i=1}^{k} u_{i} e_{i}(t), \quad\left(t, u_{1}, u_{2}, \ldots, u_{k}\right) \in I \times \mathfrak{R}^{k}
$$

Let $M^{*}$ be a $(k+1)$-dimensional semi-ruled surface in the semi-Euclidean space $E_{v}^{n+1}$, let $\left\{e_{1}, e_{2}, \ldots, e_{k}\right\}$ be the natural companion basis of the generating space $E_{k, \mu}(s)$ and $e_{0}=\varphi_{*}\left(\frac{\partial}{\partial s}\right)$ be a vector field such that $\left\{e_{0}, e_{1}, \ldots, e_{k}\right\}$ is an orthonormal basis of $\chi\left(M^{*}\right)$. In addition, suppose that the vector field system $\left\{\xi_{k+1}, \xi_{k+2}, \ldots, \xi_{n}\right\}$ is an orthonormal basis of $T_{M^{*}}^{\perp}(p)$ at $p \in M^{*}$.

Then,

$$
\left\{e_{0}, e_{1}, \ldots, e_{k}, \xi_{k+1}, \xi_{k+2}, \ldots, \xi_{n}\right\}
$$

is an orthonormal basis of $T_{E_{V}^{n+1}}(p)$ at $p \in M^{*}$. Thus, the equations of the derivative can be written in the form

$$
\bar{D}_{e_{r}} \xi_{j}=\sum_{i=0}^{k} a_{r i}^{j} e_{i}+\sum_{i=k+1}^{n} b_{r i}^{j} \xi_{i}, \quad 0 \leq r \leq k \text { ve } k+1 \leq j \leq n
$$

Hence, the Weingarten equation can be written as

$$
\bar{D}_{e_{r}} \xi_{j}=-A_{\xi_{j}}\left(e_{r}\right)+D_{e_{r}}^{\perp} \xi_{j}, \quad k+1 \leq j \leq n .
$$

Then, from equation (13) and (14) the matrix $A_{\xi_{j}}\left(e_{r}\right) \in \chi\left(M^{*}\right)$ that coresponds to the linear mapping $A_{\xi_{j}}$ is

$$
A_{\xi_{j}}=\left[\begin{array}{ccccc}
a_{00}^{j} & a_{01}^{j} & a_{02}^{j} & \ldots & a_{0 \mathrm{k}}^{j} \\
a_{10}^{j} & a_{11}^{j} & a_{12}^{j} & \ldots & a_{1 \mathrm{k}}^{j} \\
\vdots & \vdots & \vdots & \vdots & \vdots \\
a_{k 0}^{j} & a_{k 1}^{j} & a_{k 2}^{j} & \ldots & a_{k k}^{j}
\end{array}\right]_{(k+1) \times(k+1)} \quad, \quad k+1 \leq j \leq n
$$

Also $\left\langle e_{i}, e_{h}\right\rangle=\varepsilon_{i} \delta_{i h}, \quad 1 \leq i, h \leq k$, for the orthonormal basis of the generating space $E_{k, \mu}(s)$ where 


$$
\varepsilon_{i}=\left\{\begin{array}{ll}
+1 & , 1 \leq i \leq k-\mu \\
-1 & , k-\mu+1 \leq i \leq k .
\end{array} \text { and } \mu \leq v\right.
$$

If we take the inner product of the both sides of the derivative equations (13) with $e_{h}$, we get

$$
\left\langle\bar{D}_{e_{r}} \xi_{j}, e_{h}\right\rangle=-\left\langle\bar{D}_{e_{r}} e_{h}, \xi_{j}\right\rangle=\varepsilon_{h} a_{r h}^{j}
$$

Since the vectors $e_{h}$ are parallel in $E_{v}^{n+1}, \bar{D}_{e_{r}} e_{h}=0$. Thus, we find

$$
a_{r h}^{j}=0, \quad 1 \leq r, h \leq k
$$

From (2) and (3), we get

$$
a_{h 0}^{j}=\varepsilon_{0} \varepsilon_{h} a_{0 h}^{j}
$$

for the components of $A_{\xi_{j}}$. Substituting these in (15), we get $\varepsilon_{0 i}=\varepsilon_{0} \varepsilon_{i}$ and

$$
A_{\xi_{j}}=\left[\begin{array}{ccccc}
a_{00}^{j} & a_{01}^{j} & a_{02}^{j} & \ldots & a_{0 k}^{j} \\
\varepsilon_{01} a_{10}^{j} & 0 & 0 & \ldots & 0 \\
\vdots & \vdots & \vdots & \vdots & \vdots \\
\varepsilon_{0 k} a_{k 0}^{j} & 0 & 0 & \ldots & 0
\end{array}\right]_{(k+1) \times(k+1)}
$$

where $\left\langle e_{0}, e_{0}\right\rangle=\varepsilon_{0}$. So, we have proved the following three theorems.

Theorem 1 Let $M^{*}$ be a $(k+1)$-dimensional semi-ruled surface in $E_{v}^{n+1}$. The matrix in (17) corresponding to the shape operator $A_{\xi_{j}}$ of $M^{*}$ is either symmetric or anti-symmetric depending on the index.

Theorem 2 Let $M^{*}$ be a $(k+1)$-dimensional semi-ruled surface in $E_{v}^{n+1}$ and $A_{\xi j}$ be the shape operator defined for the unit normal direction $\xi_{j}, \quad k+1 \leq j \leq n$. In this case, for $k \geq 2$

$$
\operatorname{det} A_{\xi_{j}}=0
$$


Theorem 3 Let $M^{*}$ be $a(k+1)$-dimensional semi-ruled surface in $E_{v}^{n+1}$. For every point in $M^{*}$ and for every normal direction, the Lipschitz-Killing curvature is zero for $k \geq 2$.

Now, let us observe some theorems and result on the Riemann curvature of a 2 dimensional sector of a semi-ruled surface.

Theorem 4 Let $M^{*}$ be a $(k+1)$-dimensional semi-ruled surface in $E_{v}^{n+1}$ and $\left\{e_{0}, e_{1}, . ., e_{k}\right\}$ be an orthonormal vector system at a neigbourhood of a point $p \in M^{*}$. The 2-dimensional section of $M^{*}$, spanned by the vectors $\left.\left(e_{i}\right)\right|_{p}, 1 \leq i \leq k$ and $\left(e_{0}\right) \mid p$ has Riemann curvature

$$
K\left(e_{i}, e_{0}\right)=-\varepsilon_{0 i}\left\langle\bar{D}_{e_{i}} e_{0}, \bar{D}_{e_{i}} e_{0}\right\rangle
$$

where $\varepsilon_{0 i}=\varepsilon_{0} \varepsilon_{i}$.

Proof. If we consider equations (8) and (9) where $R$ is the Riemann curvature tensor field of the semi-ruled surface $M^{*}$, we find the curvature of the section spanned by $\left\{e_{i}, e_{0}\right\}$ as defined.

We obtain the following.

Corollary 5 The Riemann curvature of the 2-dimensional section spanned by $\left(e_{i}\right) \mid p, 1 \leq i \leq k$, and vectors $\left(e_{0}\right) \mid p$ of a semi-ruled surface $M^{*}$ can be expressed in terms of the components of the matrix $A_{\xi_{j}}$ as

$$
K\left(e_{i}, e_{0}\right)=\sum_{j=k+1}^{n} \varepsilon_{0 i} \varepsilon_{j}\left(a_{0 i}^{j}\right)^{2}
$$

where

$$
\left\langle\xi_{j}, \xi_{j}\right\rangle=\varepsilon_{j}=\left\{\begin{array}{ll}
+1 & , k+1 \leq j \leq n-y \\
-1 & , n-y+1 \leq j \leq n
\end{array}, \mu+y=v .\right.
$$

Corollary 6 Let $M^{*}$ be a $(k+1)$-dimensional semi-ruled surface in $E_{v}^{n+1}$ and $\left\{e_{1}, e_{2}, \ldots, e_{k}\right\}$ be an orthonormal basis for the generating space $E_{k, \mu}(t)$. The Riemann curvature of the 2-dimensional section spanned by $\left\{e_{i}, e_{j}\right\}$ is

$$
K\left(e_{i}, e_{j}\right)=0
$$


for $1 \leq i, j \leq k$. Here we will state and prove some theorems and results on the Ricci curvature, scalar curvature and mean curvature of the $(k+1)$-dimensional semi-ruled surface in $E_{v}^{n+1}$.

Theorem 7 Let $M^{*}$ be $a(k+1)$-dimensional semi-ruled surface in $E_{v}^{n+1}$ and $\left\{e_{1}, e_{2}, . ., e_{k}\right\}$ be the orthonormal basis of the generating space $E_{k, \mu}(t)$. The Ricci curvature of $M^{*}$ in the direction of the vector fields $e_{r}, 1 \leq r \leq k$, satisfies

$$
S\left(e_{r}, e_{r}\right)=-\varepsilon_{0} \sum_{j=k+1}^{n} \varepsilon_{j}\left(a_{0 r}^{j}\right)^{2}, \quad\left\langle e_{0}, e_{0}\right\rangle=\varepsilon_{0}, \quad\left\langle\xi_{j}, \xi_{j}\right\rangle=\varepsilon_{j}
$$

Here, $a_{0 r}^{j}$ are the components of the matrix $A_{\xi_{j}}, k+1 \leq j \leq n$.

Proof. Substituting $X=Y=e_{r}, 1 \leq r \leq k$, in equation (11) and using the equation (9), we get

$$
S\left(e_{r}, e_{r}\right)=-\varepsilon_{0}\left\langle\Pi\left(e_{0}, e_{r}\right), \Pi\left(e_{0}, e_{r}\right)\right\rangle
$$

Since, $\Pi\left(e_{0}, e_{r}\right)=\Pi\left(e_{r}, e_{0}\right), \quad \bar{D}_{e_{r} e_{0}}=\Pi\left(e_{r}, e_{0}\right)$ and

$$
\bar{D}_{e_{r}} e_{0}=-\sum_{j=k+1}^{n} \varepsilon_{r} a_{0 r}^{j} \xi_{j}, \quad 1 \leq i \leq k
$$

we obtain the desired equality.

Corollary 8 The Ricci curvature of $a(k+1)$-dimensional semi-ruled surface in $M^{*}$ in $E_{\mathrm{v}}^{n+1}$ in the direction of the vector field $e_{0}$ is given by

$$
S\left(e_{0}, e_{0}\right)=\varepsilon_{0} \sum_{i=1}^{k} \varepsilon_{i} S\left(e_{i}, e_{i}\right)
$$

Theorem 9 Let $M^{*}$ be $a(k+1)$-dimensional semi-ruled surface in $E_{v}^{n+1}$ and $\left\{e_{0}, e_{1}, . ., e_{k}\right\}$ be an orthonormal basis for $\chi\left(M^{*}\right)$. Then, the scalar curvature of $M^{*}$ is

$$
r_{s k}=2 \sum_{i=1}^{k} \varepsilon_{i} S\left(e_{i}, e_{i}\right)
$$

Proof. Using the equation (12) and Corollary 2.2., we get 


$$
r_{s k}=2 \sum_{i=1}^{k} K\left(e_{0}, e_{i}\right)
$$

Here, considering the property $K\left(e_{0}, e_{i}\right)=K\left(e_{i}, e_{0}\right)$ of a sectional curvature and Corollary 2.1. and Theorem 2.6., we obtain the desired equality.

Corollary 10. Let $M^{*}$ be $a(k+1)$-dimensional semi-ruled surface in $E_{v}^{n+1}$ and $e_{0}$ the unit tangent vector of the base curve of $\mathrm{M}^{*}$. Then, the scalar curvature of $M^{*}$ is equal to $2 \varepsilon_{0}$ times the Ricci curvature in the direction of the tangent vector field $e_{0}$.

Theorem 11. Let $M^{*}$ be a $(k+1)$-dimensional semi-ruled surface in $E_{v}^{n+1}$ and $e_{0}$ the unit tangent vector of the base curve of $M^{*}$. Then, the mean curvature of $M^{*}$ is

$$
H=\frac{\varepsilon_{0}}{k+1} \Pi\left(e_{0}, e_{0}\right)
$$

Proof. We substitute $r=h=0$ in (16) and using the derivative equations (1), we get either

$$
\left\langle\bar{D} e_{0} \xi_{j}, e_{0}\right\rangle=\varepsilon_{0} a_{00}^{j}
$$

or

$$
\left\langle\bar{D} e_{0} e_{0}, \xi_{j}\right\rangle=-\varepsilon_{0} a_{00}^{j} .
$$

Considering the equations (14) and (3), we have

$$
\left\langle\Pi\left(e_{0}, e_{0}\right), \xi_{j}\right\rangle=-\varepsilon_{0} a_{00}^{j} .
$$

For the matrix $A_{\xi_{j}}$, given by the equality (17),

$$
\operatorname{tr} A_{\xi_{j}}=-a_{00}^{j}, \quad k+1 \leq j \leq n .
$$

Substituting this in the definition of $H$ in (6), we get

$$
H=\frac{\varepsilon_{0}}{k+1} \Pi\left(e_{0}, e_{0}\right)
$$




\section{REFERENCES}

1. S. Kobayashi-K. Nomizu, Foundations of Differential Geometry, Interacience Publishers a Division of John Wiley Sons, New York, Vol. I-II 1963.

2. C. Thas, Properties of Ruled Surfaces in The Euclidean Space $E^{n}$, Academia Sinica, Vol. 6, No: 1, 133-142, 1978.

3. B. O'Neill, Semi-Riemannian Geometry, Academic Press. New York, London, 1983.

4. M. Ergüt, The Drall and the Scalar Normal Curvature of $(k+1)$-Dimensional Generalised Ruled Surfaces, Commun. Fac. Sci. Ank. Series A 38, 115-125, 1989. 